\title{
Tinidazole efficacy for amebic colitis therapeutic prophylaxis in patients with de novo acute leukemia receiving intensive chemotherapy
}

\author{
Luara Luz Arana-Luna, José Luis Álvarez-Vera, José Antonio de la Peña-Celaya, \\ Verónica Mena-Zepeda, Maricela Ortiz-Zepeda, María Eugenia Espitia-Ríos, \\ Juan Manuel Pérez Zúñiga and Martha Alvarado-Ibarra* \\ Hematology Department, Centro Médico Nacional 20 de Noviembre, Instituto de Seguridad y Servicos Sociales de los Trabajadores del Estado, \\ City of Mexico, Mexico
}

\begin{abstract}
Introduction: In Mexico, seroprevalence of Entamoeba histolytica is $8.4 \%$. The intestinal amebiasis in patients with acute leukemia of novo, after the start of chemotherapy (CT) in the Hematology Service of the CMN 20 de Noviembre is 12\%, even if patients show a negative baseline coprological test. Objective: To find out if the administration of tinidazole, in patients with acute leukemia and negative coprological test, at the beginning of the CT, decreases the incidence of amoebic colitis during the induction to remission. Method: Prospective and not comparative study. Patients with de novo diagnosis of acute leukemia who initiate induction and initial coprological CT. Tinidazole was indicated, $2 \mathrm{~g} /$ day for 5 days in the first week of CT started. They were monitored until the induction was concluded and hematopoietic recovery started. Results: 38 patients, 15 women and 23 men with a mean age of 44 years (16-72), with acute lymphoblastic leukemia 19, myeloblastic 16 and promyelocytic 3. Cases without and with intestinal amebiasis were 35 and 3 , respectively. Patients with amebiasis only received tinidazole for 3 days and it was given 2 days after the CT started. Conclusion: Tinidazole, in patients with acute de novo leukemia who initiate induction CT, is effective in the prevention of intestinal amebiasis, during the induction stage, if administered at $2 \mathrm{~g} /$ day, for five days, starting on day 1 of the CT.
\end{abstract}

KEY WORDS: Tinidazole. Amebiasis. Acute leukemia. Chemotherapy.

\section{Introduction}

Overall, there are about 100 million annual cases of amebic dysentery, colitis and liver abscess, which result in 100,000 deaths. According to the World Health Organization (WHO), in 1997, amebiasis was the third cause of death from parasitic disease. In Mexico, its frequency in 2015 was 211 cases per 100,000 population, which is lower if compared to the picture 15 years ago (1,353 per 100,000); mortality has also decreased: in 1955 it was $13 \%$ and in $20014.3 \%$, according to figures of the Historical
Compendium of Vital Statistics (National Institute of Statistics and Geography [INEGI - Instituto Nacional de Geografía y Estadística]/Ministry of Health [SSA - Secretaría de Salud]). This decrease, very influenced by availability of more effective medications, has also been reported in the Mexican Institute of Social Security'.

Entamoeba histolytica is acquired when its cysts are ingested through contaminated food or water. Upon excystation, trophozoites are released in the terminal ileum and migrate to the colon to colonize the mucosa by binding oligosaccharides of host mucin with adhesin on the amoeba surface, galactose $/ \mathrm{N}$-acetyl
Correspondence:

*Martha Alvarado-lbarra

E-mail: normoblasto@gmail.com
Date of reception: 06-03-2019

Date of acceptance: 19-03-2019

DOI: 10.24875/GMM.M19000285
Gac Med Mex. 2019;155 (Suppl 1):S22-S27

Contents available at PubMed www.gacetamedicademexico.com 
galactosamine. The trophozoites remain in the lumen as commensals, multiplying by binary fission, and meet their energy needs with ingestion of the host's microflora and nutrients. Some cysts present in the descending colon are excreted by feces and allow fecal-oral transmission. In $90 \%$ of cases, amoeba infections are asymptomatic and self-limiting ${ }^{2}$.

Histological lesions occur when trophozoites break the mucosal barrier and penetrate the underlying tissue, where they secrete enzymes that cross the extracellular matrix and destroy the cells; the parasite phagocytes the tissue and cell debris (which include erythrocytes). After invasion of the mucosa and submucosa, trophozoites can enter the portal circulation and spread to the liver and other target organs ${ }^{2}$.

The components secreted by trophozoites are recognized by receptors in epithelial cells, which by means of a potentiating nuclear factor activate pro-inflammatory genes that, in interaction with macrophages, induce a protective response in the epithelium, which increases its resistance to apoptosis ${ }^{3}$. Host responses to invasive amebiasis include the release of nitric oxide by neutrophils, macrophages, monocytes and dendritic cells. Immunoglobulin binding to the amebic surface can alter trophozoite's functions, blocking adhesion to host receptors and activating the complement pathway ${ }^{4,5}$.

Amebiasis exists throughout the world; its prevalence in developing countries is much higher due to poor socioeconomic conditions and low sanitation levels. The areas with high amebic infection rates include India, Africa, Mexico, and parts of Central and South America. Seroprevalence of $E$. histolytica in a study carried out in Mexico was $8.4 \% 6$.

The factors that influence on the infection being asymptomatic or invasive include the strain, genetic susceptibility, age and immune status. General risk factors for severe disease and increased mortality include: young age, pregnancy, corticosteroid treatment, malignant diseases, malnutrition and alcoholism ${ }^{7-11}$.

The best diagnostic method is serological, including ELISA (enzyme linked immunosorbent assay) and IHA (indirect hemagglutination assay) ${ }^{12-15}$. Invasive colitis is usually treated with metronidazole, tinidazole (TNZ), omidazole, nitaxozanide and others, as sole treatment or in sequential administration ${ }^{16,17}$.

Acute leukemias (AL), lymphoblastic and nonlymphoblastic, are diseases where abnormal young cells proliferate (lymphoblasts or myeloblasts), invade extramyeloid organs and replace normal hematopoiesis. These are very serious neoplasms and have high morbidity and mortality. Its management involves immediate treatment, with high doses of combined chemotherapy (CT) in order to obtain and early remission. This initial phase of treatment is called induction.

Intensive CT and radiotherapy cause, in addition to immunosuppression and severe neutropenia, epithelial lesions: desquamation and necrosis of its cells, hypoplasia and loss of protective, cellular and immunological histological functions; in the mouth and pharynx, these lesions are accompanied by severe pain; this series of alterations is known as mucositis. It is easily recognizable in accessible areas, but often it is forgotten that it also occurs in the intestine ${ }^{18}$. All these consequences of CT facilitate the occurrence of invasive amebiasis, and are particularly observed in acute leukemia induction phase. Over last year (2015), 21 cases of amebiasis were observed in our Department during induction in 53 patients with acute leukemia (AL); seven died from causes directly attributable to parasitosis or in association with other conditions.

In a study carried out in our Department ${ }^{19}$, amebiasis was found not to be diagnosed in the stool test prior to initiating induction CT. This finding was compared with a control group of patients without $A L$, and the stool test results were the same. After CT was started, there were 7 cases (12\%) of invasive amebiasis detected.

The purpose of this study is to find out if the administration of an antiamebic agent, since the beginning of CT induction, decreases the frequency of invasive amebiasis during the period of remission induction, in patients with AL and initially negative stool tests for E. histolytica cysts or trophozoites.

\section{Method}

A prospective, non-comparative, longitudinal, open label study was conducted in patients de novo diagnosed with AL who started CT remission induction from September 2016 to July 2017 at the Hematology Department of the 20 de Noviembre National Medical Center, from the Institute of Social Security and Services of State Workers. The leukemia diagnosis was defined by the existence of $>20 \%$ of blasts, with a decrease in normal hematopoiesis, in addition to known clinical and hematological data.

The inclusion criteria were: patients de novo diagnosed with $\mathrm{AL}$, antineoplastic treatment naïve, with an adequate general condition for receiving induction $\mathrm{CT}$ 
and without organic inadequacies contraindicating it. Those who received any anti-amebic treatment in the course of the previous month, subjects with a history of intolerance to TNZ or pregnant women were not included. Subjects who died from causes other than amebiasis or who did not receive the complete TNZ treatment course were censored.

At the hospital, we have two anti-amebic drugs for the treatment of the invasive form: metronidazole and TNZ; the latter was chosen because it is administered for a shorter time and, in particular, due to the lower intensity and frequency of gastrointestinal discomfort occurrence, which would add to that caused by CT.

The following variables were recorded: gender, age, history of residence, occupation, tap water and raw vegetables consumption, diarrhea, dysentery or amebiasis, stool tests initial and final data, bacterial infection, fungal infection, neutrophils, aspartate aminotransferase (AST), alanine aminotransferase (ALT), total bilirubin, albumin, abdominal pain, diarrhea, dysentery and colonic mucosa sonogram.

When the patient was admitted, initial data were collected and TNZ was started and maintained during the first ten days of the start of CT; during follow-up, the remaining variables were recorded, and the study was considered to have ended at the conclusion of the remission-induction program, with evidence of progressive neutrophil recovery and a figure higher than $0.5 \times 10 \%$ L.

Patients who presented with dysentery and abdominal colic pain associated with the presence of $E$. histolytica trophozoites in the stool test were qualified as having amoebic colitis. All cases of amebiasis were corroborated with coproparasitoscopic tests. Microscopic identification of the parasite relied on its known morphological characteristics observed by expert chemists of the Coproparasitoscopy Section of the hospital General Laboratory.

In the first five patients, the TNZ dosage was 2 grams every 24 hours, by oral route, during three consecutive days. There were three cases of amebiasis. An amendment was introduced to the study protocol and the TNZ administration was changed to 2 grams every 24 hours, by oral route, for five consecutive days.

The CT programs used, for the different types of AL were:

- Induction CT for lymphoblastic AL:

- Day 4. Dexamethasone, $10 \mathrm{mg} / \mathrm{m}^{2} /$ day, by intravenous (IV) bolus, for four days.

- Day 0. Methotrexate, $12.5 \mathrm{mg}$, by IT route + cytarabine $50 \mathrm{mg}$ + dexamethasone $5 \mathrm{mg}$.
Daunorubicin, $120 \mathrm{mg} / \mathrm{m}^{2}$ BSA IV administered by continuous dripping for 48 hours, diluted in $500 \mathrm{ml}$ of isotonic saline.

- Day 2. Cyclophosphamide 1,200 mg/m² BSA IV bolus. Vincristine $1.5 \mathrm{mg} / \mathrm{m}^{2}$ BSA IV bolus. Repeat on days 9,16 and 23. Prednisone, $60 \mathrm{mg} / \mathrm{m}^{2}$ BSA/day, PO until day 23. Then, progressively decrease in the course of 9 days.

- Day 4. L-asparaginase, 4,000 IU/m² BSA/day IM. Continue Monday-Wednesday-Friday until consolidation conclusion.

- Induction CT for myeloblastic AL:

- Cytarabine: $100 \mathrm{mg} / \mathrm{m}^{2}$ BSA diluted to $20 \mathrm{mg} / \mathrm{mL}$ of physiological saline, continuous drip, days 1 to 7 .

- Idarubicin: $12 \mathrm{mg} / \mathrm{m}^{2} \mathrm{BSA}$ diluted to $1 \mathrm{mg} / \mathrm{mL}$ of physiological saline IV bolus (15 minutes), days 1-3.

- Induction CT for promyelocytic AL:

- Transretinoic acid (TRA), $45 \mathrm{mg} / \mathrm{m}^{2}$ BSA/day, $\mathrm{PO}$, divided in two takes, until complete remission, from day 1 (minimum 30 and maximum 90 days).

- Daunorubicin, $60 \mathrm{mg} / \mathrm{m}^{2} \mathrm{BSA} /$ day (or idarubicin $12 / \mathrm{mg} / \mathrm{m}^{2}$ BSA/day), 60-minute IV infusion (days 2, 4, 6, 8).

With either schedule, hematopoiesis recovery takes approximately four weeks.

Neutropenic colitis (NC) was considered when patients presented with $>38^{\circ} \mathrm{C}$ fever, abdominal pain (at least a value of 3 on the analog scale), neutrophils $<0.5 \times 10^{9} / \mathrm{L}$ and demonstration of colonic intestinal wall thickness increase $>4 \mathrm{~mm}$ (in transversal measurement) over more than $30 \mathrm{~mm}$ (longitudinal measurement) in any segment, by ultrasound or computed tomography, in the absence of amebiasis in the stool test.

Statistical analysis was performed using the SPSS Statistics ${ }^{\circledR}$ program, v22. Nominal and ordinal variables were described in percentages; scalar variables as the mean and standard deviation. Nominal variables comparative tests were carried out with the chisquare test, and ordinal variables were compared with Student's t-test or ANOVA. Association variables were processed with non-parametric tests.

The study was carried out in accordance with the ethics principles set forth in the Declaration of Helsinki and in compliance with Good Clinical Practice and lo$\mathrm{cal}$ and national institutional regulatory requirements.

The study was approved by the CMN 20 de Noviembre Ethics and Research Committees. All the patients 
Table 1. Data obtained at patient admission

\begin{tabular}{|l|c|}
\hline Data & Result \\
\hline Gender (F/M) & $15 / 23$ \\
\hline Age (Mean/range) & $44 / 16-72$ \\
\hline Tap water (Yes/No) & $7 / 31$ \\
\hline Raw vegetables (Yes/No) & $17 / 21$ \\
\hline B. hominis $(n)$ & 3 \\
C. parvum $(n)$ & 1 \\
\hline
\end{tabular}

Table 2. Baseline data

\begin{tabular}{l|c|}
\hline Data & Result \\
\hline Neutrophils x 10/L mean/min-max) & $33 / 0-292$ \\
\hline AST U/L (mean/min-max) & $43 / 19-143$ \\
\hline ALT U/L (mean/min-max) & $45 / 9-189$ \\
\hline DB mg/dL (mean/min-max) & $0.45 / 0.1-2.1$ \\
\hline IB mg/dL (mean/min-max) & $0.4 / 0.1-2.2$ \\
\hline Albumin g/dL (mean/min-max) & $3 / 2.5-4.6$ \\
\hline E. histolytica & 0 \\
\hline Lymphoblastic AL (n) & 19 \\
Myeloblastic AL (n) & 16 \\
\hline Promyelocytic AL (n) & 3 \\
\hline AST: aspartate aminotransferase; ALT: alanine aminotransferase; DB: direct bilirubin; \\
IB: indirect bilirubin; AL: acute leukemia.
\end{tabular}

accepted their participation by signing the informed consent form.

\section{Results}

From September 2016 to July 2017, 38 patients who met the inclusion criteria were studied. Backgrounds and basic data are shown in table 1. Most patients were males. The most usual place of residence was Mexico City $(n=19)$; the rest lived in 7 more states. Regarding their occupation, subjects were distributed as follows: 14 manual workers, 8 students, 7 professionals, 7 professionals with college degree and 2 administrative technicians. No history of diarrhea or amebiasis was found. Five patients had taken antiparasitic drugs as prophylaxis. Four recognized being alcoholics.

Baseline data are shown in table 2. In no case were amoebas found (trophozoites or cysts) in the initial tests. In extra-intestinal locations, 13 bacterial infections were identified in the upper airways. In the initial
Table 3. Final data

\begin{tabular}{|l|c|c|c|c|}
\hline Variable & Amebiasis & NC & Other & $p$ \\
\hline Abdominal pain & 1 & 7 & 2 & 0.005 \\
\hline Diarrhea & 0 & 4 & 1 & 0.02 \\
\hline Dysentery & 2 & 1 & 0 & $>0.05$ \\
\hline E. histolytica & 3 & 0 & 0 & $>0.05$ \\
\hline CMT (0.6-1.0 cm) & 3 & 7 & 0 & $>0.05$ \\
\hline Neutrophils x 10\%/L (mean) & 2.1 & 1.9 & 3.0 & $>0.05$ \\
\hline AST U/L (mean) & 62 & 98 & 43 & $>0.05$ \\
\hline ALT U/L (mean) & 64 & 65 & 59 & $>0.05$ \\
\hline DB mg/dL (mean) & 1.8 & 3.6 & 0.9 & 0.03 \\
\hline IB mg/dL (mean) & 0.7 & 1.1 & 0.7 & $>0.05$ \\
\hline Albumin g/dL (mean) & 3.3 & 2.4 & 3.0 & 0.009
\end{tabular}

AST: aspartate aminotransferase; ALT: alanine aminotransferase; DB: direct bilirubin; IB: indirect bilirubin; NC: neutropenic colitis; CMT: colonic mucosa thickness.

stool tests, two unexpected germs were reported: Blastocystis hominis and Cryptosporidium parvum.

Table 3 shows the final data. The most common type of colitis was NC, and its clinical and biochemical repercussions were greater regarding abdominal pain, diarrhea, neutropenia, direct bilirubin, albumin and intestinal mucosa thickness magnitude, when compared with the cases of amebiasis $(p<0.03)$.

There were three patients with amebiasis, all with intestinal location. All three had dysentery and abdominal pain. There were other infections: 37 patients had a respiratory tract bacterial infection. One had pulmonary mycosis. They were included in a febrile neutropenia and antifungal therapeutic program.

Five patients received TNZ for three days; two had amebiasis. The rest, according to the amendment, took it for five days; one had amebiasis ( $p=0.04)$. In the three patients with amebiasis, metronidazole was used as an alternative treatment. All of them healed.

In 29 cases, TNZ was started the same day CT was initiated; no one had amebiasis. In 9 patients, it was started between 3 and 7 days after CT was initiated; the three amebiasis cases occurred in this setting $(p=0.0001)$; in two of them, administration was for 3 days.

There were eight deaths reported $(21 \%)$, with the causes being: hemorrhage in three, NC in two, febrile neutropenia in one, pulmonary mycosis in one and ATRA syndrome in one.

There was no statistical significance in the univariate and multivariate analyses, in the possible association between alcoholism, tap water intake, raw vegetables 
consumption and occupation and the occurrence of amebiasis. There was also no relationship with the type of leukemia and indicated CT.

\section{Discussion}

In terms of age, gender and history, the study population has characteristics that do not distinguish it from the general population. In all patients, the result of the initial stool test was negative, similar to the results already reported in the previous study by the Hematology Department ${ }^{19}$.

The negative results in the stool test support the idea that cysts are found in the intestinal mucosa and that they are not detected ${ }^{12-15}$. In our study, fecal matter was only examined on one occasion, which has a sensitivity not higher than $50 \%$ and that increases if the test is repeated three times in a period no longer than ten days ${ }^{20}$. In our patients, delaying CT administration for that long was not possible.

The WHO recommends for the diagnosis of intestinal amebiasis to be established with E. histolytica-specific molecular tests or with immunological techniques ${ }^{20}$. Some of these tests allow amebic antigens or antibodies determination in fecal matter or serum by different techniques, with sensitivity and specificity of 90 to $100 \%$. We found logistical problems to perform these studies. In addition, the immunological type demonstrates current existence of amebiasis or its presence years back ${ }^{14,21}$. Finally, we found no evidence of amebiasis, with support on clinical data or on stool tests, before starting $\mathrm{CT}$, but we did afterwards with the same test plus the coproparasitoscopic analysis, and with associated clinical data, absent in other varieties, which are considered commensal organisms: Entamoeba dispar, Entamoeba moshkovski and Entamoeba bangladeshi.

We found no bibliographical references in Pubmed during the last ten years that allowed us compare our results.

TNZ usual dose, for three days, was not effective in the cases herein studied. Failures disappeared when the administration was prolonged to 5 days, if administration was started on CT day 1. On the other hand, if TNZ was started after CT day 2, amebiasis occurred in some cases. Probably, if TNZ is started on day 1 , prolonging it for more than 3 days is not necessary. These data indicate the virulence of the amoeba and the few defenses of patients with AL receiving intensive CT. Administration for 5 days was not associated with adverse effects; possibly, they were masked by those of CT. Metronidazole is the anti-amebic that is regarded as the most effective for invasive amebiasis; however, standard-dose TNZ is not reported as being less active ${ }^{22}$. In the cases of amebiasis observed in this study, treatment was switched to metronidazole because of the easiness of its endovenous application.

Most patients with intestinal disorders had NC. Their manifestations were more intense than in amebiasis, with statistical significance for abdominal pain, direct bilirubin increase and albumin decrease. These differences are related to the magnitude of intestinal mucosa lesions and NC longer evolution time. In international guidelines for the treatment of intestinal infectious complications in cancer patients, it is common to find recommendations regarding $\mathrm{NC}^{23,24}$. Conversely, no recommendations are mentioned for invasive amebiasis. It is necessary that, in countries with high incidence and prevalence of amebiasis, protocols are developed aimed at the treatment of amebiasis in patients with cancer, particularly if they receive intensive $\mathrm{CT}$.

The most common causes of death were hemorrhage and NC. Mortality associated with the latter is consistent with other published reports ${ }^{16,25}$. There was no death from amebiasis, which is a relevant point in support to indicating therapeutic prophylaxis with TNZ to patients like those herein presented.

\section{Conclusions}

The absence of amoebas in fecal matter in patients with $\mathrm{AL}$ that will initiate remission-induction intensive CT does not mean the absence of invasive amebiasis once CT is started, even if there is no history suggestive of this parasitosis. The suppression of histological, cellular and immune defenses in the host, caused by the effect of CT, explain E. histolytica aggressive behavior.

The administration of TNZ for five days at a dose of $2 \mathrm{~g} /$ day PO and started at the time CT is initiated, prevents the onset of invasive amebiasis in these patients. This Indication is applicable in patients who are to undergo hematopoietic cell transplantation.

\section{References}

1. Trevino Garcia-Manzo N, Escandon-Romero C, Escobedo de la Pena J, Hernandez-Ramos JM, Fierro-Hernandez H. Amebiasis in the epidemiologic transition in Mexico: its morbidity and mortality trends in the Mexican Institute of Social Security. Arch Med Res. 1994;25:393-9.

2. Mortimer L, Chadee K. The immunopathogenesis of Entamoeba histolytica. Exp Parasitol. 2010;126:366-80 
3. Stanley SL Jr. Amoebiasis. Lancet. 2003;361:1025-34.

4. Cornick S, Tawiah A, Chadee K. Roles and regulation of the mucus barrier in the gut. Tissue barriers. 2015;3:e982426.

5. Stanley SL Jr. Protective immunity to amebiasis: new insights and new challenges. J Infect Dis. 2001;184:504-6.

6. Caballero-Salcedo A, Viveros-Rogel M, Salvatierra B, Tapia-Conyer R, Sepulveda-Amor J, Gutierrez G, et al. Seroepidemiology of amebiasis in Mexico. Am J Trop Med Hyg. 1994;50:412-9.

7. Haque R, Huston CD, Hughes M, Houpt E, Petri WA. Amebiasis. 2003;348:1565-73.

8. Petri WA Jr, Singh U. Diagnosis and management of amebiasis. Clin Infect Dis. 1999;29:1117-25.

9. Ximenez C, Cerritos R, Rojas L, Dolabella S, Moran P, Shibayama M, et al. Human amebiasis: breaking the paradigm? Int J Environ Res Public Health. 2010;7:1105-20.

10. Baxt LA, Singh U. New insights into Entamoeba histolytica pathogenesis. Curr Opin Infect Dis. 2008;21:489-94.

11. Bercu TE, Petri WA, Behm BW. Amebic colitis: New insights into pathogenesis and treatment. Curr Gastroenterol Rep. 2007;9:429-33.

12. Wilson IW, Weedall GD, Hall N. Host-parasite interactions in Entamoeba histolytica and Entamoeba dispar: what have we learned from their genomes? Parasite Immunol. 2012;34:90-9.

13. Morf L, Singh U. Entamoeba histolytica: a snapshot of current research and methods for genetic analysis. Curr Opin Microbiol. 2012;15:469-75.

14. Tanyuksel M, Petri WA, Jr. Laboratory diagnosis of amebiasis. Clin Microbiol Rev. 2003;16:713-29.

15. Skappak C, Akierman S, Belga S, Novak K, Chadee K, Urbanski SJ, et al. Invasive amoebiasis: a review of Entamoeba infections highlighted with case reports. Can J Gastroenterol Hepatol. 2014;28:355-9.
16. Baden LR, Swaminathan S, Angarone M, Blouin G, Camins BC, Casper C, et al. Prevention and Treatment of Cancer-Related Infections, Version 2.2016, NCCN Clinical Practice Guidelines in Oncology. Journal of the National Comprehensive Cancer Network: JNCCN. 2016;14:882-913.

17. Bernin $\mathrm{H}$, Marggraff $\mathrm{C}$, Jacobs $\mathrm{T}$, Brattig N, Le VA, Blessmann J, et al. Immune markers characteristic for asymptomatically infected and diseased Entamoeba histolytica individuals and their relation to sex. BMC infectious diseases. 2014;14:621.

18. Keefe DM. Intestinal mucositis: mechanisms and management. Current opinion in oncology. 2007;19:323-7.

19. Castellanos Galan JE. Amebiasis intestinal en pacientes con leucemia aguda de novo. CMN 20 de Noviembre ISSSTE. UNAM, 2001.

20. Fotedar R, Stark D, Beebe N, Marriott D, Ellis J, Harkness J. Laboratory diagnostic techniques for Entamoeba species. Clinical microbiology reviews. 2007;20:511-32.

21. Singh A, Houpt E, Petri WA. Rapid diagnosis of intestinal parasitic protozoa, with a focus on Entamoeba histolytica. Interdisciplinary perspectives on infectious diseases 2009;2009:547090.

22. Dans LF, Martínez EG. Amoebic dysentery. BMJ clinical evidence. 2007; 2007:918.

23. Freifeld AG, Bow EJ, Sepkowitz KA, Boeckh MJ, Ito JI, Mullen CA, et al. Clinical practice guideline for the use of antimicrobial agents in neutropenic patients with cancer: 2010 update by the infectious diseases society of america. Clinical infectious diseases: an official publication of the Infectious Diseases Society of America. 2011;52:e56-93.

24. Guerrant RL, Osterholm MT, Bennish ML, Pickering LK, Slutsker L Griffin PM, et al. Practice guidelines for the management of infectious diarrhea. Clinical Infectious Diseases. 2001;32:331-51.

25. Davila ML. Neutropenic enterocolitis. Current opinion in gastroenterology. 2006;22:44-7. 